\title{
Integration of aquablation through telemetry: an alternative to onsite proctoring?
}

\author{
Jose M. El-Asmar ${ }^{1}\left[\right.$ D $\cdot$ Muhieddine Labban ${ }^{1}\left(\mathbb{D} \cdot\right.$ Albert El-Hajj $^{1}$
}

Received: 17 September 2020 / Accepted: 15 January 2021 / Published online: 6 February 2021

(c) The Author(s), under exclusive licence to Springer-Verlag GmbH, DE part of Springer Nature 2021

\begin{abstract}
Purpose To evaluate the feasibility of telementoring for aquablation by comparing the outcomes of onsite versus telemetry proctoring.

Methods The telemetry device of choice was Proximie, an innovative digital platform that uses live video stream with an augmented reality technology. Our study retrospectively reviewed outcomes from our IRB approved prospective Aquablation database from March 2018 till October 2019. Procedures were guided by a proctor either onsite or remotely through telemetry. One-way ANOVA or Chi-square was used to compare perioperative parameters and mixed model ANOVA was used to compare functional outcomes.

Results Our data included 59 patients who underwent a proctored-based Aquablation of which 21 were telementor guided and 38 were onsite guided. The initial ten procedures were done with the latter approach. There was no statistical difference in age, comorbidities, prostate size, and baseline serum markers amongst the two groups. In contrast, telementor guidance was associated with increased general anaesthesia use (76.2\% vs. $21.1 \%)$ and haemostatic cauterization (81.0\% versus $47.4 \%)$ with a $p$ value $<0.00001$ and 0.004 respectively. However, the main procedure outcomes: operative time, time to Foley catheter removal, haemoglobin drop, urinary retention, and adverse events were statistically insignificant ( $p$ value $>0.05$ ). Conclusion Following an initial phase of onsite proctoring, telementoring can be safely used in the adoption phase of a new robotic technology. This approach allowed more flexibility in patient scheduling and reduced travel costs with similar surgical outcomes.
\end{abstract}

Keywords Benign prostatic hyperplasia $\cdot$ Remote consultation $\cdot$ Robotics $\cdot$ Telemedicine $\cdot$ Technology

\section{Introduction}

Telemedicine is the virtual conveyance of healthcarerelated information between two sites [1]. Telementoring, a branch of telemedicine, includes teleproctoring,

Jose M. El-Asmar and Muhieddine Labban have equally contributed to the manuscript.

Albert El-Hajj

ae67@aub.edu.lb

Jose M. El-Asmar

je56@aub.edu.lb

Muhieddine Labban

m135@aub.edu.lb

1 Division of Urology, Department of Surgery, American University of Beirut Medical Center, Riad El-Solh, Beirut 1107 2020, Lebanon telestration, and tele-assistance. Teleproctoring involves real-time remote guidance of trainees or practicing physicians to acquire new skills [1]. Telestration, the drawing on remote monitors, and tele-assistance, the remote control of the camera and instruments, are additional features that could enhance the learning experience [2]. In contrast, telesurgery is the conduction of the operation remotely. The highlight of telemetry is augmented reality (AR) or the ability to superimpose a live three-dimensional (3D) representation of the operating room setting and the patient's internal organs with the virtual world through audio-visual accompaniment and telestration [3]. One of the earliest examples of robotic telementoring was conducted between Baltimore and Munich in 2002 during a laparoscopic renal cyst ablation [4]. Additionally, the recent widespread adoption of laparoscopic and robotically assisted surgeries allows 3D image projection on a monitor that can be shared in real-time for feedback 
[2]. As such, telemetry assistance was introduced to the teaching of more complex urological surgeries including laparoscopic heminephrectomy, bladder augmentation, and ureteral lithotomy [2].

Telesurgical telementoring can be used in the remote training of surgeons especially while acquiring the skills needed to independently perform a novel procedure or new operative techniques $[5,6]$. Telesurgical telementoring has proven effective in areas of conflict like Gaza [3]. Thus, it could be of great use during the coronavirus (COVID-19) pandemic when traveling is impossible and social distancing is required [7]. In this paper, we sought to evaluate the feasibility of telesurgical telementoring for aquablation, a novel robotic-assisted treatment modality for benign prostatic obstruction (BPO), by comparing the surgical and functional outcomes of onsite versus telemetry-assisted procedures.

\section{Patients and methods}

The study retrospectively reviewed surgical and functional outcomes from our institutional review board (IRB) approved prospectively filled aquablation database between March 2018 and October 2019. The procedures were consecutive and guided, onsite or remotely through telemetry, by a total of three proctors interchangeably depending on their availability. The first ten cases were performed by an onsite proctor in order to ensure familiarization of the setup, equipment, and steps of the procedure. For the following cases, the allocation to onsite or remote proctoring was solely dependent on the physical availability of the proctor at our institution. The telemetry device of choice was Proximie (Fig. 1). Proximie is a secure platform and abides by general data protection regulation (GDPR) and Health Insurance Portability and Accountability Act (HIPAA) regulations.

Baseline demographics, comorbidities, basic metabolic serum markers, and BPO specific variables such as prostate size and preoperative prostate-specific antigen (PSA) serum levels were compared between onsite mentored and telemetry-assisted procedures. The type of anesthesia used (general versus spinal anesthesia) and whether selective cautery was used at the bladder neck for hemostasis were included. To evaluate for the effect of telementoring on the surgical outcomes, the operative time (minutes), length of hospital stay (days), time to foley removal (hours), haemoglobin drop (g/ $\mathrm{dL}$ ), percent (\%) drop in PSA, the rate of urinary retention, and 30-day adverse event were compared between the two groups. The 3-month functional outcomes were also examined including drop in American Urological Association's international prostate symptom score (IPSS), quality of life assessed by a single question, and post-void residue (PVR).

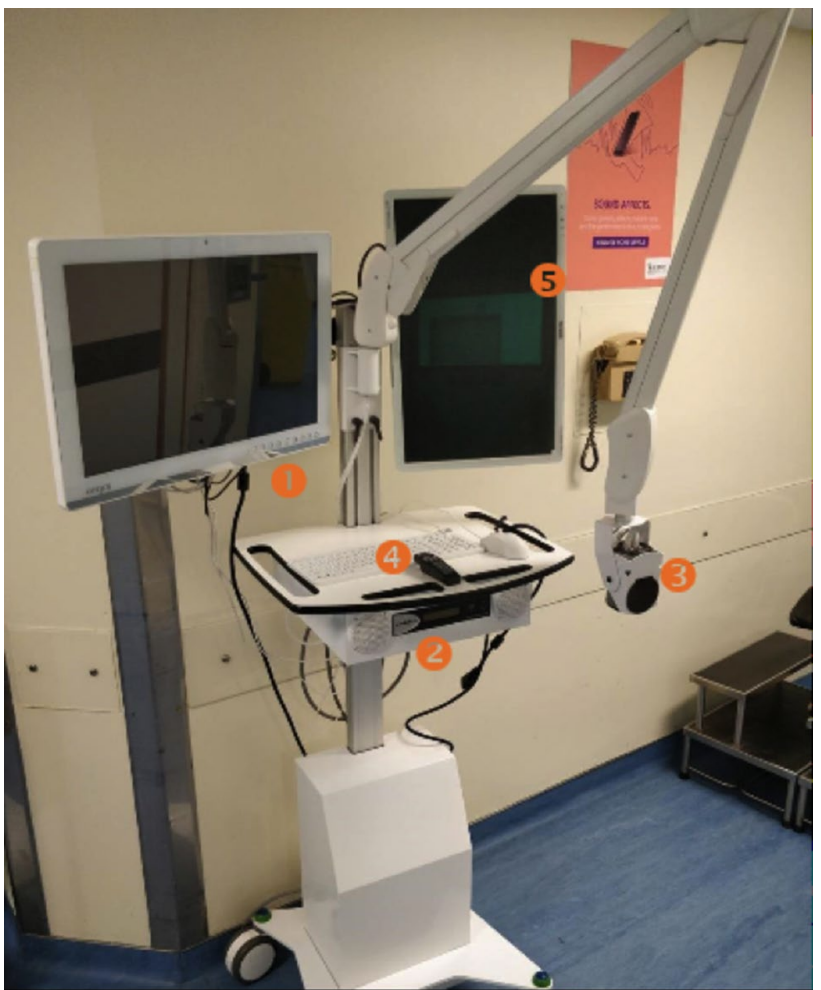

Fig. 1 Proximie platform. 1. Main computer system 2. Audio/Video System 3. Camera with optical zoom 4. Remote Control 5. Secondary screen

\section{Setup}

Proximie is an innovative digital platform that uses AR live video stream. The user-friendly application can transmit up to four feeds simultaneously at HD quality. It can support up to 40 participants who can virtually scrub to assist, annotate, and train surgeons. By offering the remote surgeon a bird's eye view of the procedure, guidance and feedback is possible between the operating and remotely assisting surgeons [3]. It uses low bandwidth internet connectivity (2-3 Megabits per second) for the video feed and runs in the cloud through Google Chrome and on a highly secure encrypted Amazon Web server. It enables a one-way stream video from the operating room to the proctoring site through a robotic camera with optical zoom features that can be moved by remote control. In addition, a twoway audio is used for effective communication between the mentor and their trainee; whereas, two real-time display screens form the interface for telestration (Fig. 1). Proximie can connect to several devices simultaneously including video camera, fluoroscopy, endoscope, and even surgical lights.

Figure 2 illustrates the Proximie's integration and setup during aquablation. Aquablation is a novel surgical 


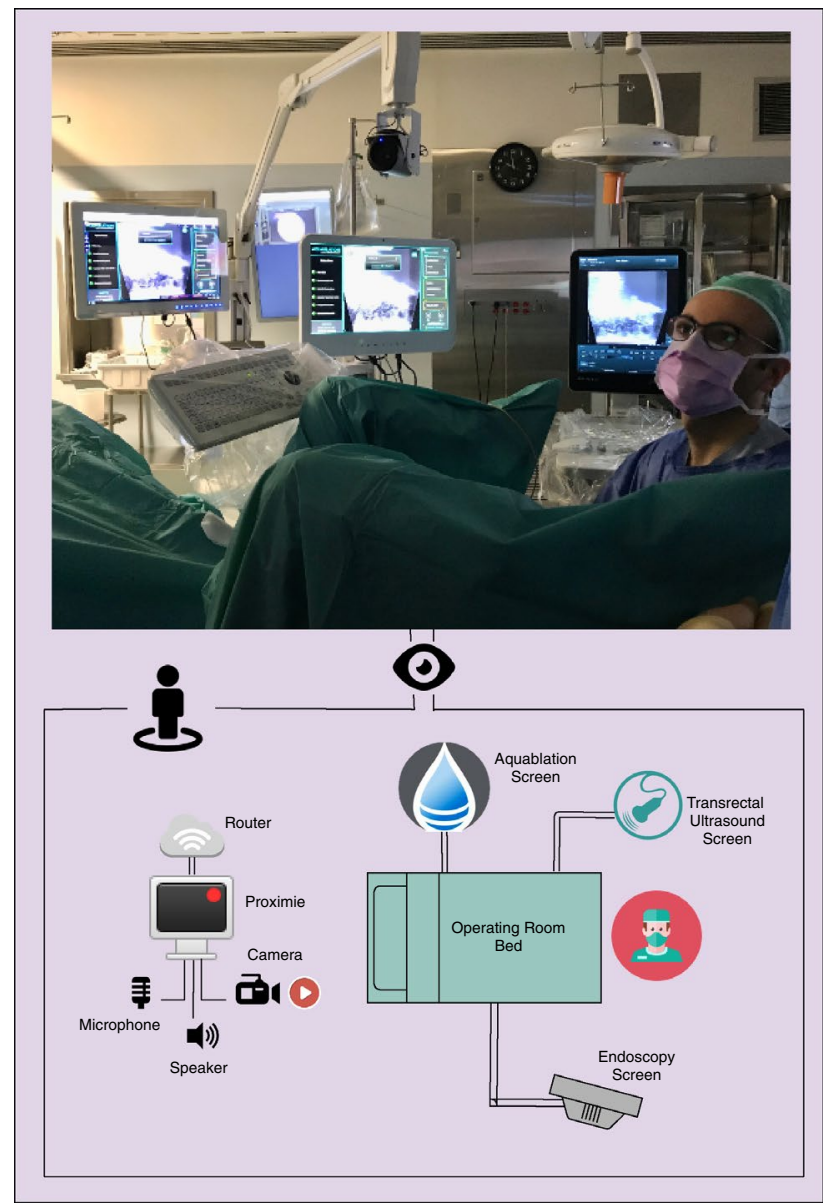

Fig. 2 Setup of Telemetry assistance during aquablation

technique that relies on real-time ultrasound feedback to visualize the prostatic tissue [8]. After demarcation of the area to be resected, a semi-automated ablation of the prostate is carried out using high-pressure water [8]. During aquablation, Proximie can be easily incorporated onto the surgical field allowing proctors to gain, through their tablets, full audio-visual access to the operative steps. In addition, they can provide the necessary input through a variety of augmented reality tools such as a digital pen for telestration or a virtual hand that superimposes on the surgical video feed creating a real-time collaboration into a 3D interface connecting the two distant sites [3]. In the adoption phase of aquablation, the remote surgeon can supervise and provide guidance, by teleproctoring or by telestration, during crucial steps of the procedure such as the mapping of the resection area and the methods to preserve the verumontanum. These factors directly impact the patients' functional outcome including voiding improvement and ejaculatory preservation.

\section{Statistics}

One-way ANOVA test was used for continuous variables whereas Chi-squared or Fisher's exact test was used for categorical variables. Mixed model ANOVA was used to evaluate the postoperative change in prostate size, PSA, haemoglobin, IPSS, and QOL in the function of the method of assistance provided (onsite assistance versus teleproctoring). The Statistical Package for the Social Sciences IBM SPSS version 24 (IBM Corp., Armonk, N.Y., USA) was used for conducting the statistics and a $p$ value of $>0.05$ was considered significant.

\section{Results}

Fifty-nine patients underwent a proctored based aquablation of which 21 (35.6\%) were telemetry guided and 38 (64.4\%) were onsite guided (Table 1). The median procedure ranks were 39 for the telemetry group and 21.5 for the onsite guided group. In order to familiarize with the setup and
Table 1 Baseline demographics, comorbities, and serum markers for patients undergoing aquablation factored by on-site proctoring versus telemetry assisted procedures

\begin{tabular}{lllc}
\hline Variable & On-site Proctoring $(n=38)$ & Telementoring $(n=21)$ & $P$ value \\
& $N(\%) ; x \pm \mathrm{SD}($ Median $)$ & $N(\%) ; x \pm \mathrm{SD} /$ (Median) & \\
\hline Age & $69.4 \pm 7.7(69)$ & $66.2 \pm 8.0(64)$ & 0.072 \\
BMI & $28.3 \pm 4.2(27.2)$ & $27.3 \pm 3.4(26.6)$ & 0.419 \\
ASA $\geq 2$ & $31(81.6 \%)$ & $15(71.4 \%)$ & 0.513 \\
General anesthesia & $8(21.1 \%)$ & $16(76.2 \%)$ & $<0.0001$ \\
INR & $1.0 \pm 0.1(1.0)$ & $1.0 \pm 0.1(1.0)$ & 0.071 \\
Hemoglobin & $14.4 \pm 1.4(14.4)$ & $14.4 \pm 1.4(14.8)$ & 0.791 \\
PSA & $4.2 \pm 4.3(3.1)$ & $4.2 \pm 2.7(3.8)$ & 0.313 \\
Prostate size $(\mathrm{g})$ & $71.1 \pm 36.5(62.1)$ & $71.8 \pm 19.8(73.7)$ & 0.605 \\
In retention & $8(21.1 \%)$ & $6(28.6 \%)$ & 0.538 \\
Cautery use & $18(47.4 \%)$ & $17(81.0 \%)$ & 0.012 \\
\hline
\end{tabular}

ASA American Society of Anesthesiology class; INR international normalized ratio; PSA Prostate Specific Antigen 
the new surgical technology, the first ten procedures were onsite guided. Subsequently, the surgeon did not allocate the patients, but the approach was solely dependent on the availability of the proctor. The average age of patients who underwent on-site proctored aquablation $(69.4 \pm 7.7)$ was congruent to the age of patients who underwent a telemetry-guided procedure $(66.2 \pm 8.0 ; p=0.072)$. Additionally, there was no statistical difference in the American Society of Anesthesiology class $\geq 2(p>0.05)$. Similarly, the average preoperative haemoglobin and international normalized ratio (INR) were similar between the two groups $(p=0.791$ and 0.071 ). The baseline patient serum PSA and prostate weight were $4.2 \pm 4.3 \mathrm{ng} / \mathrm{dL}$ and $71.1 \pm 36.5 \mathrm{~g}$ for the on-site proctored procedures and $4.2 \pm 2.7 \mathrm{ng} / \mathrm{dL}$ and $71.8 \pm 19.8 \mathrm{~g}$ for the telemetry guided surgeries $(p=0.313$ and 0.605 , respectively). In contrast, the group with telemetry guidance was associated with increased general anaesthesia use (76.2\% versus $21.1 \%)$ and hemostatic cauterization at the bladder neck ( $81.0 \%$ versus $47.4 \%)(p<0.0001$ and 0.012 , respectively).

The operative time was $49.0 \pm 20.8$ and $47.7 \pm 16.1 \mathrm{~min}$ for onsite and telemetry-assisted procedures, respectively $(p=0.824)$ (Table 2). There was also no significant difference in either the length of hospital stay or catheterization time ( $p=0.662$ and 0.473 , respectively). The rates of prior to discharge catheterization, re-hospitalization, and 3-month adverse events were 13.2, 2.6, and $23.7 \%$ for the onsite proctored procedures versus $19.0,14.3$, and $42.9 \%$ for the telemetry group ( $p>0.05$ for all variables). Most of the complications were to grade I and grade II adverse events:
75 and $87.5 \%$ for the onsite and telemetry groups, respectively (Table 2).

Figure 3 depicts the change in prostate size, PSA, hemoglobin, IPSS, QOL, and PVR factored by proctoring type. There was a large effect of surgery on haemoglobin drop, prostate size, IPSS, and QOL irrespective of proctoring type $(p<0.0001$ for all variables). There was also a drop in serum PSA and PVR ( $p=0.004$ and 0.044 , respectively). Nevertheless, the type of proctoring did not impact any of the surgical or functional outcomes $(p>0.05)$.

\section{Discussion}

In this paper, we compared the surgical and functional outcomes of aquablation among telemetry-assisted and onsite proctoring procedures. We demonstrated that there is no surgical or functional difference between the two groups. Telemetry guided procedures had a greater median rank due to the fact that several of the initial procedures were onsite guided and performed on the same day to accommodate for the proctor's availability. These numbers reflect the concept where novel surgical tools and techniques could be effectively incorporated using remote proctoring after the direct help of an onsite mentor. The economic burden and prolonged time spent during travelling to specialized centres for courses, demonstrations, and hands-on experience during the adoption phase of novel technology such as aquablation might deter urologists. However, telesurgery telementoring bridges this distance and offers
Table 2 Comparison of perioperative outcomes in aquablation between on-site proctering versus telementory assistance

\begin{tabular}{llll}
\hline Perioperative surgical outcomes & On-site Proctoring $(n=38)$ & Telementoring $(n=21)$ & $p$ value \\
& $N(\%) ; x \pm$ SD (Median) & $N(\%) ; x \pm$ SD (Median) & \\
\hline Operative time (min) & $49.0 \pm 20.8(47.5)$ & $47.7 \pm 16.1(46.0)$ & 0.824 \\
Length of stay (days) & $2.2 \pm 1.0(2.0)$ & $2.2 \pm 0.8(2.0)$ & 0.662 \\
Length of catheterization (days) & $1.7 \pm 1.1(2.0)$ & $2.8 \pm 4.2(2.0)$ & 0.473 \\
Prior to discharge recatheterization & $5(13.2 \%)$ & $4(19.0 \%)$ & 0.708 \\
Re-hospitalization & $1(2.6 \%)$ & $2(9.5 \%)$ & 0.218 \\
3-month adverse events & $8(21.1 \%)$ & $9(42.9 \%)$ & 0.08 \\
Clavien-Dindo Grade I & & $3(37.5 \%)$ & \\
Acute urinary retention & $2(25.0 \%)$ & $2(25.0 \%)$ & \\
Overactive bladder & $1(12.5 \%)$ & - & \\
Hemorrhoid thrombosis & $1(12.5 \%)$ & $1(12.5 \%)$ & \\
Clavien-Dindo Grade II & & $1(12.5 \%)$ & \\
Urinary tract infection & $2(25.0 \%)$ & $1(12.5 \%)$ & \\
Deep vein thrombosis & - & $1(12.5 \%)$ & \\
Capsular perforation & - & & \\
Clavien-Dindo Grade IIIa & & & \\
Decreased urinary flow & $1(12.5 \%)$ & & \\
Clavien-Dindo Grade IIIb & $1(12.5 \%)$ & & \\
Urethral stricture & & & \\
\hline
\end{tabular}




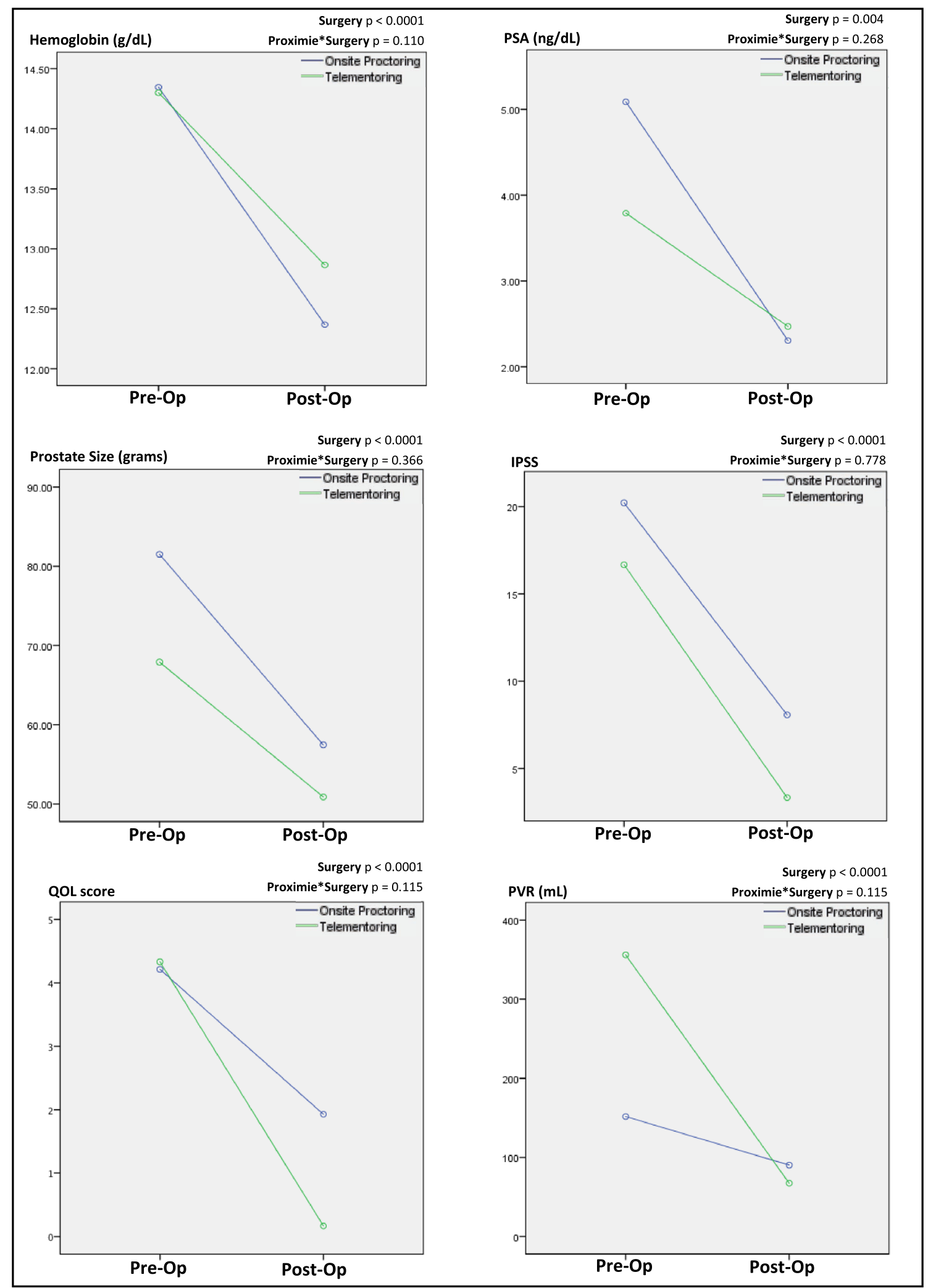

Fig. 3 Comparison of functional outcomes between onsite and telemetry proctored procedures 
the surgeon professional guidance and feedback on every patient. Additionally, telementry-assisted surgeries prove to reduce the surgeon's learning curve, especially that traditional courses are often insufficient to independently perform the surgeries [9]. As such, telementoring increases the surgeon's confidence level and minimizes complications during the surgical procedure. On the other hand, patients have indirect access to world-renowned authorities and experts in the field [2].

Shin et al. evaluated the surgical outcomes and the residents' performance during the basic steps of robotic-assisted nephrectomy and prostatectomy in onsite versus telemetryassisted surgery [10]. There was neither a difference in the perioperative variables (operative time and amount of blood loss) nor in the residents' self-assessment of their performance [10]. However, the mentors preferred remote to onsite guidance [10]. Similarly, according to our results, telementored telesurgery can be effectively and safely used for minimally invasive technologies similar to aquablation after a hands-on experience guided by an onsite mentor [11]. Our first ten aquablation surgeries were onsite mentored and were aimed at providing the initial steps of the procedure and the familiarization with the equipment. Nevertheless, the power of telementoring lies in its ability to abridge the transition between a mentor-dependent phase and full selfreliance. As such, the suggested platform strengthens previously learned skills during onsite mentoring and ensures safety of care. Effectively, with more experience, the role of the proctor transitions into an advisory assistant to perfect surgical and functional outcomes.

Our data reveal that there was no difference in major perioperative parameters between onsite and tele-assisted mentoring except for the proportion of general anesthesia and cautery used for hemostasis, which were more common among telementored cases. During the initial telemetry sessions, some patients were uncomfortable with the echoing discussion between the surgeon and the mentor. Consequently, based on our experience and the patients' feedback the following patients were offered the chance to opt for general anesthesia to avoid noise or elicited anxiety. Furthermore, cautery use was significantly higher in telementored cases because most of these cases where performed after the issuance of updated recommendations on aquablation revealing that selective bladder neck cauterization reduces transfusion rates [12].

Telementoring enables real-time feedback by an expert despite them being miles away. Such a commodity would reduce cost associated with travelling and logistics expenses [13]. In the wake of the recent COVID-19 pandemic, the integration of telecommunication platforms, like Proximie, is more essential than ever to mitigate risks associated with preventable exposures [14]. Similarly, the decentralization of knowledge and skills would be possible, as large educational centers would collaborate with sister hospitals as well as hospitals across the globe.

Telementoring is yet to attain a complete acceptance within the urological community. At the time, there are several barriers that need to be addressed. Platform liability, additional costs, changes in workflow, patient acceptance, and third-party coverage of telemetry-related costs are among the barriers faced with telementoring implementation $[15,16]$. However, the regulation of telemetry by urological associations would endorse the widespread acceptance among both urologists and their patients.

\section{Conclusion}

This paper shows that telementoring can be adopted by surgeons when learning new surgical techniques. Given the recent travel impact caused by the COVID-19 pandemic, telementoring has become almost a necessity for self-development. Until randomized trials are available to attest telemontoring's efficacy and safety for novel surgical techniques, we suggest a blended training program including initial onsite assistance transitioning into telementoring.

Author contributions EAJ: design; writing-original draft; visuals, interpretation of data; data acquisition LM: writing — original draft; statistics; interpretation of data EHA: conceptualization, writing —editing and review, supervision. All authors approved the final version of the manuscript to be published and agreed to be accountable for all aspects of the work in ensuring that questions related to the accuracy or integrity of any part of the work are appropriately investigated and resolved.

Funding Not applicable.

Data availability The data is the private property of the American University of Beirut Medical Center (AUBMC). Data can be shared if requested from the principal investigator.

\section{Compliance with ethical standards}

Conflict of interest The authors declare that they have no conflict of interest.

Ethics approval The study was approved by the institutional review board at the American University of Beirut (SUR.AE.03).

Consent to participate Oral consent was obtained from patients.

\section{References}

1. Lee BR, Bishoff JT, Janetschek G et al (1998) A novel method of surgical instruction: international telementoring. World J Urol 16(6):367-370. https://doi.org/10.1007/s003450050082 (published Online First: Epub Date) 
2. Challacombe B, Kavoussi L, Patriciu A, Stoianovici D, Dasgupta P (2006) Technology Insight: telementoring and telesurgery in urology. Nat Clin Pract Urol 3(11):611-617. https://doi. org/10.1038/ncpuro0626 (published Online First: Epub Date)

3. Greenfield MJ, Luck J, Billingsley ML et al (2018) Demonstration of the effectiveness of augmented reality telesurgery in complex hand reconstruction in Gaza. Plast Reconstr Surg Glob Open 6(3):e1708-e1808. https://doi.org/10.1097/GOX.0000000000 001708 (published Online First: Epub Date)

4. Frimberger D, Kavoussi L, Stoianovici D et al (2002) Telerobotic surgery between Baltimore and Munich. Der Urologe. Ausg. A 41(5):489-492. https://doi.org/10.1007/s00120-002-0197-z (published Online First: Epub Date)

5. Bove P, Stoianovici D, Micali S et al (2003) Is telesurgery a new reality? Our experience with laparoscopic and percutaneous procedures. J Endourol 17(3):137-142. https://doi.org/10.1089/08927 7903321618699 (published Online First: Epub Date)

6. Lee BR, Moore R (2000) International telementoring: a feasible method of instruction. World J Urol 18(4):296-298. https://doi. org/10.1007/s003450000136 (published Online First: Epub Date)

7. Ghebreyesus DTA. WHO Director-General's opening remarks at the media briefing on COVID-19-11 March 2020. Secondary WHO Director-General's opening remarks at the media briefing on COVID-19-11 March 2020 [speech] 2020. WHO DirectorGeneral's opening remarks at the media briefing on COVID-1911 March 2020.

8. Gilling P, Barber N, Bidair M et al (2018) WATER: a double-

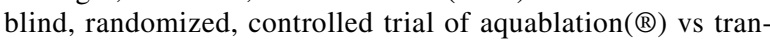
surethral resection of the prostate in benign prostatic hyperplasia. J Urol 199(5):1252-1261. https://doi.org/10.1016/j. juro.2017.12.065 (published Online First: Epub Date)

9. Hinata N, Miyake H, Kurahashi T et al (2014) Novel telementoring system for robot-assisted radical prostatectomy: impact on the learning curve. Urology 83(5):1088-1092. https://doi. org/10.1016/j.urology.2014.01.010 (published Online First: Epub Date)
10. Shin DH, Dalag L, Azhar RA et al (2015) A novel interface for the telementoring of robotic surgery. BJU Int 116(2):302-308. https:// doi.org/10.1111/bju.12985 (published Online First: Epub Date)

11. Bruschi M, Micali S, Porpiglia F et al (2005) Laparoscopic telementored adrenalectomy: the Italian experience. Surg Endosc 19(6):836-840. https://doi.org/10.1007/s00464-004-9124-2 (published Online First: Epub Date)

12. Elterman D, Bach T, Rijo E et al (2020) Transfusion rates after 800 Aquablation procedures using various haemostasis methods. BJU Int. https://doi.org/10.1111/bju.14990 (published Online First: Epub Date)

13. Sen V, Aydogdu O, Yonguc T, Bozkurt IH, Bolat D (2016) Telerounding \& telementoring for urological procedures. Archivio italiano di urologia, andrologia : organo ufficiale [di] Societa italiana di ecografia urologica e nefrologica 88(3):206-207. https:// doi.org/10.4081/aiua.2016.3.206 (published Online First: Epub Date)

14. Vaidya R, Herten-Crabb A, Spencer J, Moon S, Lillywhite L (2020) Travel restrictions and infectious disease outbreaks. J Travel Med 27(3):taaa050. https://doi.org/10.1093/jtm/taaa050 (published Online First: Epub Date)

15. Ellimoottil C, Skolarus T, Gettman M et al (2016) Telemedicine in urology: state of the art. Urology 94:10-16. https://doi. org/10.1016/j.urology.2016.02.061 (published Online First: Epub Date)

16. Castaneda P, Ellimoottil C (2019) Current use of telehealth in urology: a review. World J Urol. https://doi.org/10.1007/s0034 5-019-02882-9 (published Online First: Epub Date)

Publisher's Note Springer Nature remains neutral with regard to jurisdictional claims in published maps and institutional affiliations. 